Artículo

\title{
Reducción del desarrollo de la roya anaranjada del café con mezcla de homeopáticos
}

\author{
Cesáreo Rodríguez-Hernández ${ }^{1 \S}$ \\ Sandra Ramos-Reyes ${ }^{2}$ \\ Esteban Escamilla-Prado ${ }^{3}$ \\ Felipe de Jesús Ruiz-Espinoza ${ }^{2}$
}

${ }^{1}$ Entomología y Acarología-Colegio de Posgraduados. Carretera México-Texcoco km 36.5, Montecillo, Texcoco, Estado de México. CP. 56230. Tel. 595 9517374. ${ }^{2}$ Universidad Autónoma Chapingo. Carretera México-Texcoco km 38.5, Chapingo, Estado de México. CP. 56230. Tel. 5959579658 (sann.ramos@hotmail.com; agrohomeopatia@hotmail.com). ${ }^{3}$ Centro Regional Universitario OrienteUniversidad Autónoma Chapingo. Carretera Huatusco-Xalapa km 6.5, Huatusco, Veracruz. CP. 94100. Tel. 55 61934846. (espreschoca@yahoo.com.mx).

${ }^{\S}$ Autor para correspondencia: crhernan@ colpos.mx.

\section{Resumen}

La roya anaranjada (Hemileia vastatrix Berk. \& Broome) es la principal enfermedad del cultivo del café, y ante la necesidad de disminuir las pérdidas que ocasiona, se han implementado alternativas ecológicas para su control y bajo este concepto esta investigación tuvo como objetivo cuantificar el efecto de cuatro preparados homeopáticos y caldo bordelés al $0.5 \%$ en la severidad, infestación foliar y defoliación causadas por este hongo, el experimento se realizó en el Centro Regional de Oriente de la Universidad Autónoma Chapingo en Huatusco, Veracruz, México, de septiembre a noviembre de 2014, donde se asperjaron los homeopáticos cada semana durante 60 d, contando con cuatro repeticiones/árboles por tratamiento en un diseño de bloques completos al azar. El caldo bordelés al $0.5 \%$ se aplicó dos veces durante $60 \mathrm{~d}$ y a los árboles testigos no se les realizó ninguna aplicación. A los 60 d se registró la severidad en 10 hojas por árbol y a los 30 y 60 d se cuantificó la infestación foliar y la defoliación. Los resultados mostraron que no se afectó la severidad a los 60 d ni la infestación foliar a los 30 d, el polifármaco de plantas (mezcla de 72 preparados homeopáticos antiestresantes y de enfermedades, nutrientes, plagas y plantas de importancia agrícola)/212 Centesimal Hahnemanniana, disminuyó 49.75\% la defoliación a los 30 d y la mezcla de homeopáticos redujo 26.65 y $45.06 \%$ la infestación foliar y defoliación a los 60 d. La mezcla de homeopáticos permite proteger a la planta más que manejar o eliminar al patógeno, es una alternativa ecológica y orgánica para resolver la contingencia actual de la roya del café.

Palabras clave: Hemileia vastatrix, agrohomeopatía, alternativas, biorracional.

Recibido: junio de 2019

Aceptado: agosto de 2019 


\section{Introducción}

El cultivo de café (Coffea arabica L.) ha sido afectado por la roya anaranjada en los últimos siete años, provocando un desequilibrio ecológico y económico en Chiapas, Oaxaca, Puebla y Veracruz, México, principalmente (Avelino y Rivas, 2014). Es un hongo que invade la hoja y causa defoliación del árbol, disminuyendo el rendimiento y la calidad física y sensorial del grano (Medina-Meléndez et al., 2016).

El control se ha realizado principalmente con la aplicación de caldo bordelés, oxicloruro de cobre y fungicidas organosintéticos sistémicos, como cyproconazol (Lutz, 2012); sin embargo, existe una demanda creciente de la sociedad de producir alimentos sanos sin comprometer el entorno, lo que obliga a buscar alternativas ecológicas que no afecten al ambiente, al cultivo y que la cosecha sea inocua, no afecten la salud y economía del productor, que eviten la dependencia tecnológica y que propicien la autosuficiencia tecnológica.

Entre varias alternativas ha destacado la homeopatía (Ruiz, 2015), como una opción fitosanitaria para manejar el hongo y propiciar mejor aptitud biológica en el cultivo. La mezcla de arsénico blanco, azufre y tierra de silicio a la 6 Centesimal Hahnemanniana $(\mathrm{CH})$ y nosode (preparado homeopático del mismo problema fitosanitario que se desea controlar) a la $30 \mathrm{CH}$, aplicada cuatro veces al follaje, disminuye las manchas de roya, pero además induce crecimiento en los ápices, mejora la estructura vegetativa y reduce la defoliación (Leonel y Barros, 2013).

La aspersión quincenal de yoduro de potasio a la $200 \mathrm{CH}$ y nosode a la 200 y a la $204 \mathrm{CH}$ inhibe en 18.79-77.16, 13.39-58.28\% y 17.6-44.18, respectivamente, el desarrollo de pústulas de la roya de 1 a 2.5 meses, de acuerdo con Rodríguez-Hernández et al. (2014), quienes además señalan que la aplicación del nosode a la 6, 7 y $8 \mathrm{CH}$ protege a las hojas y se recuperan los cafetales a los $30 \mathrm{~d}$ de la aspersión en Simojovel, Chiapas. No obstante, no se ha evaluado el efecto de los productos homeopáticos en la severidad y en la infestación foliar de la roya ni en la defoliación del cultivo.

Esta investigación tuvo como objetivo evaluar la aspersión de cuatro preparados homeopáticos y caldo bordelés al $0.5 \%$ al follaje y su efecto en el desarrollo de la roya anaranjada del café y en la defoliación, mediante la cuantificación de la severidad, infestación foliar y hojas caídas, después de cuatro y ocho aplicaciones de los homeopáticos y de dos aspersiones de caldo bordelés.

\section{Materiales y métodos}

Este experimento se realizó de septiembre a noviembre del 2014 en las parcelas experimentales del Centro Regional de Oriente (CRUO) de la Universidad Autónoma Chapingo (UACH), en Huatusco, Veracruz, México, dentro de una plantación comercial de cafetos en etapa productiva de la variedad Garnica, donde se seleccionaron 24 plantas de 10 años de edad, con $65.46 \%$ de infestación foliar de roya anaranjada en una superficie de $2700 \mathrm{~m}^{2}$, con $90 \mathrm{~m}$ de largo y $30 \mathrm{~m}$ de ancho, sobre una ladera bajo el sistema de cultivo con sombra en niveles inferiores a $40 \%$, donde las principales labores culturales que se han realizado cada año han sido: podas en el mes de agosto, fertilización una vez al año con estiércol fresco de bovino, deshierbe y aplicación de caldo bordelés dos veces al año. 
Los homeopáticos evaluados fueron: nosode de roya H. vastatrix a la $200 \mathrm{CH}$ (Nos-roya $200 \mathrm{CH}$ ), polifármaco de café (combinación de 12 preparados homeopáticos, de 1 especie de ácaro, 5 de hongos, 4 de insectos, 1 de liquen y 1 de nematodo) a la $200 \mathrm{CH}(\mathrm{PFC} 200 \mathrm{CH})$, polifármaco de plantas (mezcla de 72 preparados homeopáticos de importancia agrícola, donde se integraron 4 especies de ácaros, 6 antiestresantes, 4 especies de bacterias, 1 de crustáceo terrestre, 12 de hongos, 19 de insectos, 2 de moluscos, 1 de nematodo, 20 nutrientes y 3 especies de plantas) a la $212 \mathrm{CH}$ (PFP/212 CH) y la mezcla de éstos.

El Nos-roya $200 \mathrm{CH}$ se elaboró con el hongo $H$. vastatrix, colectado de hojas de café con presencia de pústulas en el mes de octubre de 2013 en la comunidad de Jalapilla, Xicotepec de Juárez, Puebla, México; ubicada a $500 \mathrm{msnm}$ en las coordenadas de longitud $20^{\circ} 22^{\prime} 22^{\prime \prime}$ y latitud $-97^{\circ} 54^{\prime} 54^{\prime \prime}$. El PFC $200 \mathrm{CH}$ es una mezcla de los preparados homeopáticos de diversas especies de ácaros, hongos, insectos, liquen y nematodos, a la $200 \mathrm{CH}$ cada uno, cuya descripción de su contenido se específica en Ruiz (2015). El PFP/212 CH contiene las dinamizaciones de bacterias, hongos, insectos, ácaros, moluscos, nematodos y de plantas como chile (Capsicum annuum L.), quimafila (Chimaphila umbellata (L.) WPC Barton) y mercadela (Calendula officinalis L.) a la $200 \mathrm{CH}$, además de antiestresantes y elementos mayores y menores a la $204 \mathrm{CH}$ (Ruiz, 2015).

De cada preparado homeopático, se utilizó $0.03 \mathrm{~mL}$ y se diluyó en $1 \mathrm{~L}$ de agua contenida en un recipiente de plástico de $1.5 \mathrm{~L}$ y después de sucusionar, agitar el líquido con movimientos ascendentes y descendentes en forma contundente y continua por 2 min, se mezcló en una mochila aspersora con $4 \mathrm{~L}$ de agua. En la mezcla de homeopáticos se usaron $0.03 \mathrm{~mL}$ de Nos-roya $200 \mathrm{CH}$, PFC 200 CH y de PFP/212 CH, y se diluyeron en 1 L de agua, luego se sucusionó, diluyó y asperjó.

En la preparación del caldo bordelés al $0.5 \%$ (c. bordelés $0.5 \%$ ), se diluyeron $50 \mathrm{~g}$ de sulfato de cobre en $5 \mathrm{~L}$ de agua y se incorporó a $50 \mathrm{~g}$ de cal hidratada diluidos en $5 \mathrm{~L}$ de agua y se asperjó al follaje. La aplicación se realizó al follaje de 24 plantas seleccionadas al azar en cuatro bloques, que representaron cuatro repeticiones. Los homeopáticos se asperjaron ocho veces con frecuencia semanal durante $60 \mathrm{~d}$, iniciando el 8 de septiembre y terminando el 30 de octubre, y el c. bordelés $0.5 \%$ se aplicó dos veces durante los 60 d; el 8 y 18 de septiembre.

En cada planta se seleccionaron tres ramas, de los estratos inferior, medio y superior, y en las ramas superiores e inferiores se marcaron tres hojas y en el estrato medio se marcaron cuatro, teniéndose 10 hojas marcadas por árbol, 240 en todo el experimento. A éstas, previo a la primera aplicación, $1 \mathrm{~d}$ antes, se les cuantificó severidad, infestación foliar y defoliación. La severidad se midió a los 0 y 60 d mediante la escala de daño de la SENASICA (2013), la cual va del 0 al 6; indicando $0=$ sin síntomas visibles, $1=$ apenas se observan puntos cloróticos, 2, 3, 4, 5 y 6 cuando hay 2, 7, 20, $45 \mathrm{y} \geq 70 \%$ de área foliar afectada por el hongo, respectivamente.

La severidad inicial se multiplicó por 100 y se dividió entre la severidad final y después de restarle 100 se obtuvo el desarrollo neto de la roya en porcentaje en ese periodo de tiempo. La infestación foliar (número de hojas con roya anaranjada en la rama) se determinó a los 0, 30 y 60 d mediante el conteo de hojas con presencia de pústulas, el cual se multiplicó por 100 y se dividió entre el total de hojas en cada rama seleccionada para un porcentaje, como lo menciona CENICAFE (Montes et al., 2011). En seguida, la infestación inicial se multiplicó por 100 y se dividió entre la infestación final (a los 30 y 60 d) y luego de restarle 100 se obtuvo el incremento neto de la infestación, en porcentaje para los 30 y $60 \mathrm{~d}$. 
La defoliación consistió en contar las hojas prendidas en cada rama seleccionada a los 0, 30 y 60 d y en comparación a la lectura inicial se calculó el porcentaje de defoliación; el número de hojas a los 30 y 60 d se multiplicó por 100 y se dividió entre el número de hojas iniciales, y se le restó 100 para obtener el porcentaje de defoliación para ese tiempo.

A los porcentajes de desarrollo de severidad, de infestación foliar y a la defoliación, se les realizaron las pruebas de los supuestos experimentales referentes a homogeneidad de varianzas con la prueba Levenne (Steel y Torrie, 1986) y normalidad de los errores con la prueba modificada de Shapiro Wilks (Royston, 1982), con niveles de significancia de $p \leq 0.05$ y el análisis de varianza se efectuó con la prueba no paramétrica de Friedman (Márquez, 2008), realizando comparaciones múltiples de los promedios de los rangos con el programa InfoStat (Balzarini et al., 2008), versión 2015.

\section{Resultados y discusión}

La severidad a los 60 d después de la aplicación de los tratamientos se observa en la Cuadro1, donde se constata que el experimento se inició con un promedio de $13.1 \%$ dentro del rango de 10.93 a $18.24 \%$ (junto con el testigo) y al término de este tiempo el promedio aumentó a $60.17 \%$, con variación de 57.33 a $62.95 \%$, esto indica que el incremento promedio de la severidad a los 60 d fue de $47.07 \%$ con respecto a la fecha inicial, y específicamente de $48.62 \%$ en el testigo, que es el incremento real de severidad en el experimento.

Cuadro 1. Severidad de $H$. vastatrix en el cultivo de café a los 60 d de la aplicación de los tratamientos.

\begin{tabular}{|c|c|c|c|}
\hline \multirow{2}{*}{ Tratamientos } & \multicolumn{2}{|c|}{ Severidad (\%) } & Desarrollo \\
\hline & Inicial & $60 \mathrm{~d}$ & $60 \mathrm{~d}$ \\
\hline C. bordelés $0.5 \%$ & 10.93 & 57.6 & $(88.18)^{*} 4.75^{* *} b$ \\
\hline Nos-roya $200 \mathrm{CH}$ & 11.61 & 61.65 & (81.7) $2.75 \mathrm{ab}$ \\
\hline Mezcla homeopáticos & 12.02 & 58.55 & (73.85) $1.75 \mathrm{a}$ \\
\hline PFC $200 \mathrm{CH}$ & 11.48 & 62.95 & (88.87) $4.75 \mathrm{~b}$ \\
\hline $\mathrm{PFP} / 212 \mathrm{CH}$ & 18.24 & 57.33 & (77.34) $3 \mathrm{ab}$ \\
\hline Testigo & 14.33 & 62.95 & (84.12) $4 \mathrm{ab}$ \\
\hline
\end{tabular}

${ }^{*}=$ media de los tratamientos en porcentaje $;{ }^{* *}=$ media de rangos. Promedios de rangos con la misma letra en desarrollo son similares estadísticamente en su efecto con la prueba de Friedman.

El avance de la severidad no fue homogéneo, debido a que se inició con distinta intensidad y a que hubo diferente efecto de los tratamientos. El desarrollo normal de la enfermedad, observado en el testigo de $84.12 \%$ a los 60 d, fue similar estadísticamente a la de los tratamientos; sin embargo, c. bordelés $0.5 \%$ y PFC $200 \mathrm{CH}$ incrementaron en 4.82 y $5.64 \%$ la severidad y con la mezcla de homeopáticos se redujo $12.2 \%$ el desarrollo de la severidad normal.

De esta manera, se observa que cuando las hojas de café presentan de $10.93 \%$ a $18.24 \%$ de severidad en septiembre, como en las condiciones de Huatusco, Veracruz, los productos homeopáticos y el c. bordelés $0.5 \%$ no detienen el desarrollo de la severidad de la roya en noviembre, a los $60 \mathrm{~d}$, tiempo en el cual solamente reducen $12.2 \%$ el avance de ésta, como el caso de la mezcla de homeopáticos aplicada cada $8 \mathrm{~d}$ por dos meses. 
La infestación foliar de la roya, número de hojas infestadas del total de hojas en la rama muestreada, registrada a los 30 y 60 d después de la aplicación de los tratamientos se observa en el Cuadro 2, donde se muestra que al inicio del experimento ésta fue de $65.46 \%$ en promedio, con variación de 59.14 a $74.55 \%$ entre los tratamientos, y a los 30 y 60 d el promedio de la infestación foliar fue de $89.53(83.42-98.36)$ y $87.08(72.12-98.33) \%$, respectivamente. Con esto se comprueba que la infestación se incrementó en 24.07 y $21.62 \%$ a los 30 y 60 d, en tanto que el testigo, la infestación se incrementó en 21.03 y $30.98 \%$ en los mismos tiempos, respecto al promedio inicial.

Cuadro 2. Infestación foliar causada por $H$. vastatrix en el cultivo de café a los 30 y 60 d después de la primera aplicación de homeopáticos y c. bordelés $0.5 \%$.

\begin{tabular}{ccccccc}
\hline \multirow{2}{*}{ Tratamientos } & \multicolumn{3}{c}{ Infestación $(\%)$} & & \multicolumn{2}{c}{ Desarrollo (\%) } \\
\cline { 2 - 3 } \cline { 6 - 7 } & Inicial & $30 \mathrm{~d}$ & $60 \mathrm{~d}$ & & $30 \mathrm{~d}$ & $60 \mathrm{~d}$ \\
\hline C. bordelés 0.5\% & 59.14 & 98.36 & 80.73 & & $(39.93)^{*} \mathrm{~b}$ & $(10.14) 3.83^{* *} \mathrm{~b}$ \\
Nos-roya 200 CH & 64.58 & 89.6 & 94.84 & & $(28.93) \mathrm{ab}$ & $(31) 3.96 \mathrm{~b}$ \\
Mezcla homeopáticos & 74.55 & 83.42 & 72.12 & & $(8.16) \mathrm{a}$ & $(-26.12) 1.92 \mathrm{a}$ \\
PFC 200 CH & 66.14 & 85.11 & 88.21 & & $(23.26) \mathrm{ab}$ & $(25.31) 3.17 \mathrm{ab}$ \\
PFP/212 CH & 61.04 & 92.36 & 88.28 & & $(35.39) \mathrm{ab}$ & $(25.57) 4.13 \mathrm{~b}$ \\
Testigo & 67.35 & 88.38 & 98.33 & & $(21.29) \mathrm{ab}$ & $(30.84) 4 \mathrm{~b}$ \\
\hline
\end{tabular}

${ }^{*}=$ media de los tratamientos en porcentaje. ${ }^{* *}=$ media de rangos. Promedios de rangos con la misma letra en Desarrollo son similares estadísticamente en su efecto con la prueba de Friedman.

El testigo no siempre tuvo la mayor infestación y el tratamiento que empezó con mayor infestación no fue el más dañado, por el contrario, tuvo la menor infestación.

El desarrollo de la infestación foliar a los 30 d de la aplicación no tuvo diferencia estadística con el testigo; no obstante, se incrementó hasta en $87.55 \%$ con c. bordelés $0.5 \%$ y se redujo $61.67 \%$ con la aplicación de la mezcla de homeopáticos. A los 60 d, la infestación se incrementó 30.98\%, como se observa en el testigo, el cual fue estadísticamente similar a c. bordelés $0.5 \%$, Nos-roya $200 \mathrm{CH}, \mathrm{PFC} 200 \mathrm{CH}$ y PFP/212 CH, pero disminuyó significativamente $26.65 \%$ con la aplicación de la mezcla de homeopáticos.

La infestación foliar disminuyó a los 60 d, respecto a los 30 d, en c. bordelés $0.5 \%$, mezcla de homeopáticos y PFP/212 CH debido a que se indujo formación de hojas y que bajó la proporción de las infestadas; no obstante, estas hojas jóvenes se infestaron en c. bordelés $0.5 \%$ y PFP/212 CH y se incrementó nuevamente el porcentaje de infestación, no mostrando significancia, a diferencia de las plantas de café asperjadas con la mezcla de homeopáticos, donde las hojas nuevas no tuvieron la misma infestación, por ende el porcentaje de infestación foliar se redujo hasta mostrarse negativo.

La defoliación registrada a los 30 y 60 d después de la aplicación de los tratamientos se muestra en el Cuadro 3, donde se evidencia que a los 30 d ésta fue de $32.03 \%$ en promedio y osciló de 9.6 a $56.98 \%$. Todos los tratamientos redujeron la defoliación, siendo el mejor PFP/212 CH, con 49.75\% respecto al testigo. A los $60 \mathrm{~d}$ del inicio del experimento el promedio de defoliación fue de 56.04\%, con variación de 41.27 a $75.11 \%$, obteniéndose disminución en todos los tratamientos, siendo mejor la mezcla de homeopáticos, la cual redujo en $45.06 \%$ la defoliación respecto al testigo. 
Cuadro 3. Defoliación (\%) en el cultivo de café a los 30 y 60 d después de la primera aplicación de homeopáticos y c. bordelés $0.5 \%$.

\begin{tabular}{ccc}
\hline Tratamientos & $30 \mathrm{~d}$ & $60 \mathrm{~d}$ \\
\hline C. bordelés $0.5 \%$ & $(43.93)^{*} 3.75^{* *} \mathrm{ab}$ & $(55.78) 3.13 \mathrm{ab}$ \\
Nos-roya $200 \mathrm{CH}^{\mathrm{I}}$ & $(9.60) 3.67 \mathrm{ab}$ & $(42.12) 3.88 \mathrm{ab}$ \\
Mezcla homeopáticos & $(18.68) 3 \mathrm{ab}$ & $(41.27) 2.46 \mathrm{a}$ \\
PFC 200 CH & $(34.68) 3.25 \mathrm{ab}$ & $(59.16) 3.38 \mathrm{ab}$ \\
PFP/212 CH & $(28.35) 2.88 \mathrm{a}$ & $(62.83) 3.71 \mathrm{ab}$ \\
Testigo & $(56.98) 4.46 \mathrm{~b}$ & $(75.11) 4.46 \mathrm{~b}$ \\
\hline
\end{tabular}

${ }^{*}=$ media de los tratamientos en porcentaje; ${ }^{* *}=$ media de rangos. Promedios de los rangos con la misma letra a los 30 y 60 d son similares estadísticamente en su efecto con la prueba de Friedman.

La mezcla de homeopáticos redujo la defoliación debido a que el árbol con este tratamiento retuvo hojas, en comparación al testigo, incluso en aquellas con alta severidad, las hojas se cayeron, pero la planta emitió hojas nuevas en mayor cantidad que otros tratamientos que también formaron hojas, como el caso de c. bordelés $0.5 \%$ y PFP/212 $\mathrm{CH}$ pero que no mostraron significancia estadística.

En los árboles donde se aplicó el Nos-roya 200 CH el número de hojas iniciales fue menor, y la proporción de hojas caídas con las nuevas hojas resultó menor al 50\%, lo que denotó poca recuperación. La aplicación de la mezcla de homeopáticos cada 8 d durante 60 d disminuyó el desarrollo de la severidad de la roya anaranjada del café a los 60 d (aunque no significativamente), el daño en las hojas fue menor y al no dañarlas la planta se fortaleció, se indujo resistencia al café y redujo la infestación (sin diferencia estadística a los 30 d y con significancia a los 60 d), ya que el nuevo follaje no presentó pústulas anaranjadas y a su vez, disminuyó la defoliación a los 60 d, pues las hojas más afectadas se cayeron y solo se mantuvieron en la rama aquellas donde el daño no fue tan severo, de esta forma, ayudó a que la planta se recuperará más rápido, estimulando la generación de nuevas hojas, lo que propició mayor fotosíntesis que se reflejó en el rendimiento, produciendo mayor carga y de seguir así, la aplicación del tratamiento hasta la cosecha se esperarían mejores resultados.

Existen otras mezclas de homeopáticos que también han mostrado efecto en el control de insectos y patógenos. Rodríguez-Hernández et al. (2014) afirman que al utilizarse el nosode de la roya de café $H$. vastatrix a la $3 \mathrm{CH}$ mezclado con Hypothenemus hampei (Ferrari) y sulfato de sodio a la 3 $\mathrm{CH}$, hay un control sobre la roya anaranjada y la broca de café (H. hampei). Leonel y Barros (2013) señalan que la mezcla de arsénico blanco, azufre y tierra de silicio a la $6 \mathrm{CH}$ más roya de café $H$. vastatrix a la $30 \mathrm{CH}$ reduce la defoliación en café debido a una mayor brotación de hojas.

El desarrollo de hojas al final del tratamiento, como se observó con la mezcla de homeopáticos, es un indicador de que la homeopatía fortalece a la planta, como también lo ha indicado Tichavský (2009) en tomate, con un efecto indirecto y diverso en el patógeno. Una muestra de ello es el uso de PFP/212 CH que con cuatro aspersiones en 30 d indujo más follaje; no obstante, se observaron puntos cloróticos y pústulas anaranjadas en las hojas, lo que pudo ser un indicador de que la planta se fortaleció, pero no se indujo resistencia contra el patógeno (Erdmann et al., 2011) de igual manera a los $60 \mathrm{~d}$, en el PFP/212 CH la mayor parte de las hojas iniciales se caen y se mantienen en la rama los nuevos brotes, que en su mayoría se infestan con roya. 
En este mismo periodo, él Nos-roya $200 \mathrm{CH}$ no detuvo ni incrementó la severidad, aumentó la infestación y disminuyó la defoliación, debido a que a los 30 d hubo más follaje, pero la proporción de hojas con puntos cloróticos y pústulas fue mayor; síntoma que perduró hasta los 60 d.

El mismo efecto de hojas infestadas se observó con el PFC $200 \mathrm{CH}$, donde aumentó el número de hojas con puntos cloróticos y pústulas desde los primeros $30 \mathrm{~d}$, además de incrementarse el desarrollo del daño en las hojas a los 60 d, no obteniendo los resultados esperados en comparación con Honorato (2012), quien reportó que con la utilización del polifármaco de lechuga se controlaron diversas plagas y enfermedades y se obtuvo mejor vigor y calidad en el cultivo. En café, se presentó menor recuperación de la planta y la defoliación fue en la misma proporción que las plantas donde no se realizaron aplicaciones.

Con base en lo anterior, se esperaría que con el transcurrir del tiempo las plantas asperjadas con el PFC a la $200 \mathrm{CH}$ y él Nos-roya $200 \mathrm{CH}$ tendrían mayor daño, ya que estos preparados no mostraron control sobre el hongo $H$. vastatrix, incluso el primer homeopático, estimula (sin significancia), el desarrollo de la severidad; no obstante, no es motivo para afirmar de manera general que los nosodes no funcionan; aunque con la aspersión de estos homeopáticos no se obtuvieron los resultados esperados.

Ruiz (2015) afirma que en el caso de los nosodes los síntomas pueden ser corregidos por los más similares, tomando secreciones directamente de los órganos enfermos, lo cual no se realizó de esta manera para el PFC $200 \mathrm{CH}$ y el Nos-roya $200 \mathrm{CH}$, ya que las muestras se tomaron de una parcela distinta a donde se desarrolló el experimento; para la elaboración del primer homeopático se utilizó roya de una parcela en Jalapilla, Puebla, a una altitud de $500 \mathrm{msnm}$, es posible que el material de Huatusco sea diferente y más resistente, ya que se trabajó a una altura de $1344 \mathrm{msnm}$, donde anteriormente no se desarrollaba esta enfermedad (Medina-Meléndez et al., 2016).

También se deben considerar las practicas realizadas en la parcela donde se tomó la muestra para la elaboración del homeopático, ya que de haber seleccionado hojas con roya que tuvieran un tratamiento o algún microorganismo benéfico que pudiera parasitar al hongo fitopatógeno, el nosode podría haber estimulado la supervivencia del hongo, obteniendo un efecto contrario al esperado, aunque esto es solo una hipótesis, en tal sentido se recomienda realizar el preparado para cada región y actualizarlo constantemente, debido a la rápida mutación de las razas de roya, además de utilizar hojas enfermas de plantas que no hayan sido tratadas con anterioridad.

Otra causa que se puede considerar para explicar porqué este nosodes no tienen efecto en el control del hongo, es que la potencia utilizada no haya sido la adecuada, en una investigación similar, Rodríguez-Hernández et al. (2014) reportaron que con el Nos-roya a la 6, 7 y 8 CH la planta se recupera a los $30 \mathrm{~d}$ de su aspersión, a la $204 \mathrm{CH}$ inhibe el crecimiento de la pústula, mientras que a la $200 \mathrm{CH}$ tiene poco efecto en el control del hongo.

Otros nosodes utilizados para el control de hongos que tampoco han mostrado efecto en los cultivos son el nosode de la roya del hipérico (Melampsora hypericorum (DC.) J. Schröt.) a la 30 Decimal Hahnemanniana aplicado en plantas de hipérico sin olor (Hypericum inodorum Mill.), que, aunque incrementa la enfermedad, hay menos defoliación (Erdmann et al., 2011), los nosodes de 
Alternaria solani (Ell. and Mart.) Jones and Grout. en diferentes potencias no tienen efecto sobre la germinación de esporas y el crecimiento de A. solani (Carneiro et al., 2006), lo cual se pudo deber a la influencia del medio ambiente y al genotipo de la planta.

Otro tratamiento que se utilizó fue c. bordelés $0.5 \%$, con dos aspersiones en 60 d, el cual aumentó, pero sin significancia estadística, la infestación, pues el número de hojas con presencia de pústulas fue mayor desde los primeros 30 d y a los 60 d ésta disminuyó al igual que la defoliación, debido a que las hojas nuevas y su proporción con respecto al número de hojas con pústulas fue menor; no obstante, no se detuvo el avance de la severidad, por el contrario, se observó un estímulo del daño foliar en las hojas jóvenes, mientras que con los homeopáticos apenas aparecieron unos cuantos puntos cloróticos, indicando síntomas avanzados de la enfermedad.

Los preparados homeopáticos, que también afectan a virus fitopatógenos (Rodríguez et al., 2013), estimulan la producción de metabólicos secundarios, semioquímicos que mejoran el estado fisiológico de la planta, inducen resistencia y favorecen múltiples interacciones biológicas que restablecen la energía vital de la planta (Teixeira et al., 2009) y reducen la incidencia y los daños de patógenos y plagas (Bonato, 2007).

Con base en el análisis anterior, se observa que el efecto de los preparados homeopáticos en la roya del café es variable, lo cual depende de la potencia que se use, del número de aplicaciones y de la mezcla con otros homeopáticos, además se evidencia que su efecto se observa más claro a los $60 \mathrm{~d}$ con aplicaciones semanales, donde aparentemente la roya no se elimina, pero la homeopatía permite que esta no se desarrolle normalmente, con lo que se afirma que las dinamizaciones homeopáticas tienen el potencial de reemplazar el uso de caldo bordelés, en ciertas circunstancias (Modolon et al., 2012) Teixeira, que es una de las alternativas más recurridas para la protección de café ante este patógeno (Lutz, 2012; Barrera, 2013), la homeopatía es una alternativa biorracional que no mata al hongo (Ruiz, 2015), sino que activa el sistema de resistencia en la planta, debido al estímulo de su metabolismo (Leonel y Barros, 2013).

De esta manera, la mezcla de homeopáticos disminuye la infestación y la defoliación, lo cual puede potencializarse con la aplicación de otros homeopáticos en la desintoxicación del suelo, la nutrición de la planta y otros patógenos y varias plagas o para proteger al cultivo del ataque de otros hongos, como tierra de silicio, y de ácaros, nemátodos o pulgones, como estafisagria (Meneses, 2017). Además de integrarse a un manejo cultural que conlleve podas, control de sombra y rejuvenecimiento de la plantación, entre otras medidas como biofertilización y manejo de plantas espontaneas para proteger al cultivo principalmente, evitar el ataque y daño e incidir en la proliferación del patógeno.

\section{Conclusiones}

Los productos homeopáticos, nosode de roya $H$. vastatrix, PFC $200 \mathrm{CH}$ y PFP/212 CH, y el c. bordelés $0.5 \%$, no disminuyen el desarrollo de la severidad a los $60 \mathrm{~d}$.

Ningún tratamiento disminuye el desarrollo de la infestación a los 30 d y a los 60 d los homeopáticos nosode de roya $H$. vastatrix, PFC $200 \mathrm{CH}, \mathrm{PFP} / 212 \mathrm{CH}$, y c. bordelés al $0.5 \%$ tampoco son efectivos. 
Los homeopáticos, nosode de roya $H$. vastatrix y PFC $200 \mathrm{CH}$, y c. bordelés $0.5 \%$ no reducen la defoliación a los 30 y 60 d, la aplicación del PFP/212 CH, no reduce la caída de hojas a los 60 d, no obstante, tuvo una reducción de $49.75 \%$ en la defoliación a los 30 d.

La mezcla de homeopáticos disminuye $26.65 \%$ el desarrollo de la infestación de la roya anaranjada del café y además reduce $45.06 \%$ la defoliación a los 60 d con ocho aplicaciones semanales.

\section{Literatura citada}

Avelino, J. y Rivas, G. 2014. La roya anaranjada del cafeto. https://hal.archives-ouvertes.fr/hal01071036/document. 47 p.

Balzarini, M. G.; González, L.; Tablada, M.; Casanoves, F.; Di Rienzo, J. A. y Robledo, C. W. 2008. Manual del usuario. Ed. Brujas. Grupo InfoStat, FCA. Universidad Nacional de Córdoba. http://www.infostat.com.ar Córdoba, Argentina. 82-162 pp.

Barrera, F. J. 2013. La roya del café, crónica de una devastación anunciada. Ecofronteras 49(3):22-25.

Bonato, C. M. 2007. Homeopatia em modelos vegetais. Cultura homeopática. 6(21):24-28.

Carneiro, S. M. T. P. G.; Romano, E. D. B.; Pignoni, E.; Teixeira, M. Z.; Vasconcelos, M. E. C. and Gomes, J. C. 2006. Effect of biotherapic of Alternaria solani on the early blight of tomato-plant and the in vitro development of the fungus. Inter. J. High Dilution Res. 9(33):147-155.

Erdmann, M.; Boff, M. I. C. e Boff, P. 2011. Preparados homeopáticos no manejo da ferrugem Melampsora hypericorum e da mosca branca Bemisia tabaci em plantas de Hypericum inodorum. Cadernos Agroecol. 6(2):1-5.

Honorato, M. H. 2012. Experiencias de agrohomeopatía en lechuga. In: IX Foro interinstitucional la homeopatía, la producción agropecuaria y la salud en el medio rural. Ruiz, E. F. J. (Ed.). Universidad Autónoma Chapingo. Chapingo, Texcoco, Estado de México, México. 30-33 pp.

Leonel, A. H. e Barros, B. H. R. 2013. Utilização de preparados homeopáticos para controle da ferrugem do café (Hemileia vastatrix) na região da Alta Mogiana. Cuadernos Agroecol. $8(2): 1-5$.

Lutz, G. G. 2012. Alternativas al problema de la roya del café en la República Dominicana. Consejo Nacional de Investigaciones Agropecuarias y Forestales. http://www.croplifela.org/es/ plaga-del-mes.html?id=29n San José, Costa Rica.

Márquez, L. E. 2008. Introducción a la estadística experimental. Cuarta edición. Universidad Autónoma Chapingo (UACH). Texcoco, Estado de México, México. 267 p.

Medina-Meléndez, J. A.; Ruiz-Nájera, R. E.; Gómez-Castañeda, J. C.; Sánchez-Yáñez, J. M.; Gómez-Alfaro, G. y Pinto-Molina, O. 2016. Estudio del sistema de producción de café (Coffea arabica L.). Ciencia UAT. Universidad Autónoma de Chiapas. Facultad de Ciencias Agronómicas. Villa Flores, Chiapas, México. 10(2):33-43.

Meneses, M. N. 2017. Agrohomeopatía como alternativa a los agroquímicos. Rev. Médica de Homeopatía. 10(1):9-13.

Modolon, T. A.; Boff, P.; Boff, M. I. C. e Miquelluti, D. J. 2012. Homeopathic and high dilution preparations for pest management totomato crop under organic production system. Hortic. Bras. 30(1):51-57. 
Montes, R. C.; Armando, P. O. y Amílcar, C. R. 2011. Infestación e incidencia de broca, roya y mancha de hierro en el cultivo de café del Departamento del Cauca. Biotecnología en el Sector Agropecuario y Agroindustrial. 10(1):98-108.

Rodríguez, H. C.; Cruz, B. N.; Hernández, J. C. y Ruiz, E. F. J. 2013. Homeopáticos contra virus fitopatógenos y el caso del agronosode del virus del mosaico de la calabaza. In: métodos bioracionales para el manejo de plagas. Rodríguez, H. C. y Guzmán, M. R. (Eds.). Agricultura Sostenible 8. Colegio de Postgraduados y Sociedad Mexicana de Agricultura Sostenible. Montecillo, Texcoco, Estado de México, México. 75-90 pp.

Rodríguez-Hernández, C.; Ruiz-Espinoza, F. J.; Amador-Hernández, J. A.; HernándezArizmendiz, J. C. y Martínez-Tomás, S. H. 2014. Protección del café contra la roya anaranjada Hemileia vastatrix con preparados homeopáticos. In: homeopatía: avances 2014. Ruiz, E. F. J. (Ed.). Universidad Autónoma Chapingo. Chapingo (UACH). Texcoco, Estado de México, México. 101-109 pp.

Royston, P. 1982. An extension of Shapiro and Wilk's W Test for normality to large samples. J. Royal Statistical Soc. Series C. Appl. Statistics. 31(2):115-124.

Ruiz, E. F. J. 2015. Agrohomeopatía conocimiento científico para la conservación de la vida. In: XII Foro Interinstitucional Homeopatía y Modernidad. Ruiz, E. F. J. (Ed.). Universidad Autónoma Chapingo (UACH). Chapingo, Texcoco, Estado de México, México. 30-33 pp.

SENASICA. 2013. Roya del cafeto (Hemileia vastatrix Berkeley \& Bropme). Dirección General de Sanidad Vegetal, Sistema Nacional de Vigilancia Epidemiológica Fitosanitaria, Servicio Nacional de Sanidad, Inocuidad y Calidad Agroalimentaria (SENASICA)-SAGARPA. México, DF. Ficha técnica núm. 40. 25 p.

Steel, R. y Torrie, J. 1986. Bioestadística: principios y procedimientos. 2a (Ed.). McGraw-Hill. México, DF. 622 p.

Teixeira, R.; Boff, M. I. C.; Ribeiro, L. G.; Boff, P. e Zanardi, O. Z. 2009. Efeito de preparados homeopáticos e fitoterápicos sobre Anastrepha fraterculus em condições de laboratório. Rev. Bras. Agroecol. 4(2):1429-1433.

Tichavský, R. 2009. Homeopatía para las plantas. (Ed.). Fujimoto Promociones. Monterrey, NL. México, DF. 236 p. 